\title{
Using Fuzzy Cognitive Mapping in Environmental Decision Making and Management: A Methodological Primer and an Application
}

\author{
Elpiniki Papageorgiou ${ }^{1}$ and Areti Kontogianni ${ }^{2}$ \\ 1Technological Educational Institution of Lamia, \\ Department of Informatics and Computer Technology, Lamia, \\ ${ }^{2}$ University of Aegean, Department of Marine Sciences, Mytilini, Lesvos,
}

Greece

\section{Introduction}

Widespread concerns over the integrity of natural ecosystems worldwide have initiated numerous attempts at developing new tools of monitoring present conditions, assessing future risks and visualizing alternative futures. Reports on the 'state of the world' abound and so do policy proposals and sustainability strategies. Amidst this plenty of ideas, our ability to reverse the trend and secure a safe, minimum stock of valuable natural capital seems counterproductive. A better understanding of ecosystem dynamics at both the quantitative (biochemical cycling) as well as the qualitative (ecological structure of food webs) levels, without artificial divisions between them, is needed. We also need to understand better the institutional failures leading to a growing number of 'tragedies of the commons'.

To tackle these challenges appropriately, current environmental management strategies need to 'navigate' through an apparent tension: On the one hand they must meet the demand for scientific knowledge-based policy, expressed under the motto 'science speaks to policy'. On the other hand, the very same strategies urge for stakeholder involvement and sponsor initiatives to elicit lay-people attitudes, beliefs and visions for the future. This tension seems to reflect the ever lasting stand-off of bottom up and top down approaches.

The motivation for this chapter comes from the authors' uneasiness with the present methodological arsenal in the domain of environmental stakeholder analysis. Previous research on non-market valuation of environmental assets has shown the importance of complementing the neoclassical microeconomic framework of choice in stated preference surveys with qualitative - both ex ante and ex post - analysis of individual mental processes, perceptions and beliefs (Kontogianni et al, 2001, 2005, 2008). Especially applications of contingent valuation have benefited from in-depth interviews and focus groups conducted ex ante in order for the researcher to understand the cultural, social and psychological background of choices elicited through structured interviews. (Desvousges \& Smith 1988, Brouwer, 1999). In spite though of the importance of stakeholder qualitative analysis in 
stated preferences valuation techniques, we still lack a coherent, standardized approach to analyze environmental perceptions and beliefs. The need to fill the gap becomes apparent when we recognize the fact that the way non-experts articulate complex relationships, such as those governing marine ecosystem functions, have their own special weight in influencing policy design and implementation: they transcend the fact/value divide (senso Putnam 1985) and offer valuable insights on the ways cause and effect relationships in nature are perceived (Karageorgis et al., 2006).

Fuzzy Cognitive Mapping (FCM) was thus selected as a suitable method for semiqualitative analysis to achieve our research goal. In this chapter we introduce the reader to the concept of Fuzzy Cognitive Maps (FCMs) and their theoretical background. In section 2 we summarize the state-of-the-art in qualitative, stakeholder analysis for environmental management. We then present the structure of FCMs (section 3) and the analytical use of graph theory in defining relevant indices (section 4). We proceed with the development of FCMs (section 5) and the FMC inference and simulation processes (section 6). After presenting the theoretical structure, the practical steps involved in the design and implementation of a FCM exercise are codified (section 7). We then illustrate the concepts discussed so far with a practical application implemented by the authors in The Black Sea (section 8) before we summarize and conclude in section 9.

\section{The many facets of stakeholder analysis in environmental management}

Integrated approaches to environmental planning with proper stakeholder involvement offer a possible way forward. Such an approach needs to facilitate communication within multidisciplinary research teams; it needs to recognize the functional continuity from watersheds to the coasts to the open sea, thereby helping to locate the scale of intervention less on the base of traditional jurisdictions and more towards appropriate ecosystem scales. Last but not least, it must encompass participatory management schemes which promise a substantive change in the exploitation of local knowledge. By enhancing stakeholder involvement, participatory management strengthens policy relevance, diminishes uncertainties, improves monitoring and raises enforcement rates (NRC 1996, OECD 2005).

Participatory (or deliberative) approaches to environmental management are usually grouped under the general term of stakeholder analysis (Grimble and Wellard 1997, Bryson 2004, Reed et al., 2009). Stakeholder analysis in turn can be divided into what we opt to call macro-stakeholder and micro-stakeholder analysis. The former category includes all those qualitative approaches that refer to the interaction of social groups and their dynamics: social networks analysis (Scott, 2000, Carrington et al 2005, Turnpenny et al., 2005), analysis of conflicts (Howard, 1989, Hjortso et al. 2005, 2010; Stoney \& Winstanley, 2001), and actor analysis (Hermans, 2008). The latter category refers to qualitative or semi-quantitative approaches, which explore individual perceptions, values and attitudes. These include: fuzzy cognitive mapping of social perceptions and values (Bots et al., 2000, Stone 2002), perceptions mapping (Bots, 2007), mind mapping (Buzan, 1993), concept mapping (Novak, 1993), focus groups and in-depth interviews.

Approaches in stakeholder analysis as described above share some common characteristics: they are 'eclectic but pragmatic' approaches with varying degree of sophistication, requiring in average a low in-depth academic investigation, but able to manipulate a vast quantity of soft information. Their strength lies primarily with thinking about problems than solving them. The present paper aims at contributing to a refinement of participatory management 
tools by applying fuzzy cognitive mapping (FCM) to the exploitation of local knowledge. FCM fits the requirements stated above better than any of the other conceptual modelling techniques analyzed here. Most other methods are either too difficult for the type of stakeholders we are aiming for, or take too much time. Yet, FCMs have their own set of specific disadvantages as Kok (2009) overview them.

In the case of stakeholders' analysis for ecological modeling and environmental management, the FCMs have found a good number of applications. At first, Hobbs et al., 2002 applied FCM as a tool to define management objectives for complex ecosystems (Hobbs et al., 2002). Next, Ozesmi and Ozesmi $(2003,2004)$ proposed a multi-step FCM and participatory approach of Stakeholder Group Analysis in Uluabat Lake, Turkey, for ecosystem observation. The multi-step fuzzy cognitive mapping approach analyzes how people perceive a system, and compare and contrasts the perceptions of different people or groups of stakeholders (Ozesmi \& Ozesmi, 2004).

After the pioneering work of Ozesmi \& Ozesmi (2003, 2004), in environmental and ecological management topics, other researchers followed with more implementations of FCMs in this area. FCMs have been employed in a number of studies including a FCM for rapid stakeholder and conflict assessment for natural resource management (Hjortsø et al. 2005; Robson \& Kant, 2007), a FCM for modelling a generic shallow lake ecosystem by augmenting the individual cognitive maps (Tan \& Ozesmi, 2006), FCM for predicting the effects of perturbations on ecological communities, thus to control on the fledging rate of an endangered New Zealand bird (Ramsey \& Vetman, 2005), FCM for assessing local knowledge use in agroforestry management (Isaac et al., 2009), FCM for modelling of interactions among sustainability components of an agro-ecosystem using local knowledge (Rajaran \& Das, 2009), FCM for predicting modelling a New Zealand dryland ecosystem to anticipate pest management outcomes (Ramsey \& Norbury, 2009), FCM for cotton yield management in precision agriculture (Papageorgiou et al., 2009, 2010).

\section{The structure of Fuzzy Cognitive Maps}

Fuzzy Cognitive Mapping methodology is a symbolic representation for the description and modeling of complex systems. Fuzzy Cognitive Maps (FCMs) describe different aspects of the behavior of a complex system in terms of concepts. Each concept represents a state or a characteristic of the system and interacts with each other showing the dynamics of the system. FCMs have been introduced by Kosko, (1986) as signed directed graphs for representing causal reasoning and computational inference processing, exploiting a symbolic representation for the description and modeling of a system.

In fact, FCM could be regarded as a combination of Fuzzy Logic and Neural Networks (Kosko, 1992). Graphically, FCM seems to be an oriented graph with feedback, consisting of nodes and weighted arcs. Nodes of the graph stand for the concepts that are used to describe the behavior of the system, connected by signed and weighted arcs representing the causal relationships that exist between the concepts (see Figure 1). It must be mentioned that all the values in the graph are fuzzy, so concepts take values in the range between $[0,1]$ and the weights of the arcs are in the interval [-1,1]. Observing this graphical representation it becomes clear which concept influences other concepts by showing the interconnections between them. Moreover, FCM allows updating the construction of the graph, such as the adding or deleting of an interconnection or a concept. FCMs are used to represent both 
qualitative and quantitative data. The construction of a FCM requires the input of human experience and knowledge on the system under consideration. Thus, FCMs integrate the accumulated experience and knowledge concerning the underlying causal relationships amongst factors, characteristics, and components that constitute the system.

A FCM consists of nodes or concepts, $\mathrm{C}_{\mathrm{i}}, \mathrm{i}=1 \ldots \mathrm{N}$, where $\mathrm{N}$ is the total number of concepts. Each interconnection between two concepts $C_{i}$ and $C_{j}$ has a weight, a directed edge $W_{i j}$, which is similar to the strength of the causal links between $C_{i}$ and $C_{j}$. $W_{i j}$ from concept $C_{i}$ to concept $C_{j}$ measures how much $C_{i}$ causes $C_{j}$. In simple FCMs, directional influences take on trivalent values $\{-1 ; 0 ;+1\}$, where -1 indicates a negative relationship, 0 no causal relation, and +1 a positive relationship. In general, $\mathrm{Wij}$ indicates whether the relationship between the concepts is directed or inverse. The direction of causality indicates whether the concept $C_{i}$ causes the concept $C_{j}$ or vice versa. Thus, there are three types of weights:

- $\quad W_{\mathrm{ij}}>0$ indicates a positive causality between concepts $\mathrm{Ci}$ and $\mathrm{Cj}$. That is, the increase (decrease) in the value of $\mathrm{Ci}$ leads to the increase(decrease) on the value of $\mathrm{Cj}$,

- $\quad \mathrm{W}_{\mathrm{ij}}<0$ indicates a inverse (negative) causality between concepts $\mathrm{Ci}$ and $\mathrm{Cj}$. That is, the increase (decrease) in the value of $\mathrm{Ci}$ leads to the decrease (increase) on the value of $\mathrm{Cj}$

- $\quad \mathrm{W}_{\mathrm{ij}}=0$ indicates no causality between $\mathrm{Ci}$ and $\mathrm{Cj}$.

It is important to note that $\mathrm{W}_{\mathrm{ij}} \neq \mathrm{W}_{\mathrm{ji}}$ in that causal relationship are not necessarily reversible. In Figure 1, an example FCM representation of the public health system is illustrated which has seven generic vertices $\left(C_{1}\right.$ to $\left.C_{7}\right)$ and the weights (weighted edges) showing the relationships between concepts.

\section{FCM of Public health problem}

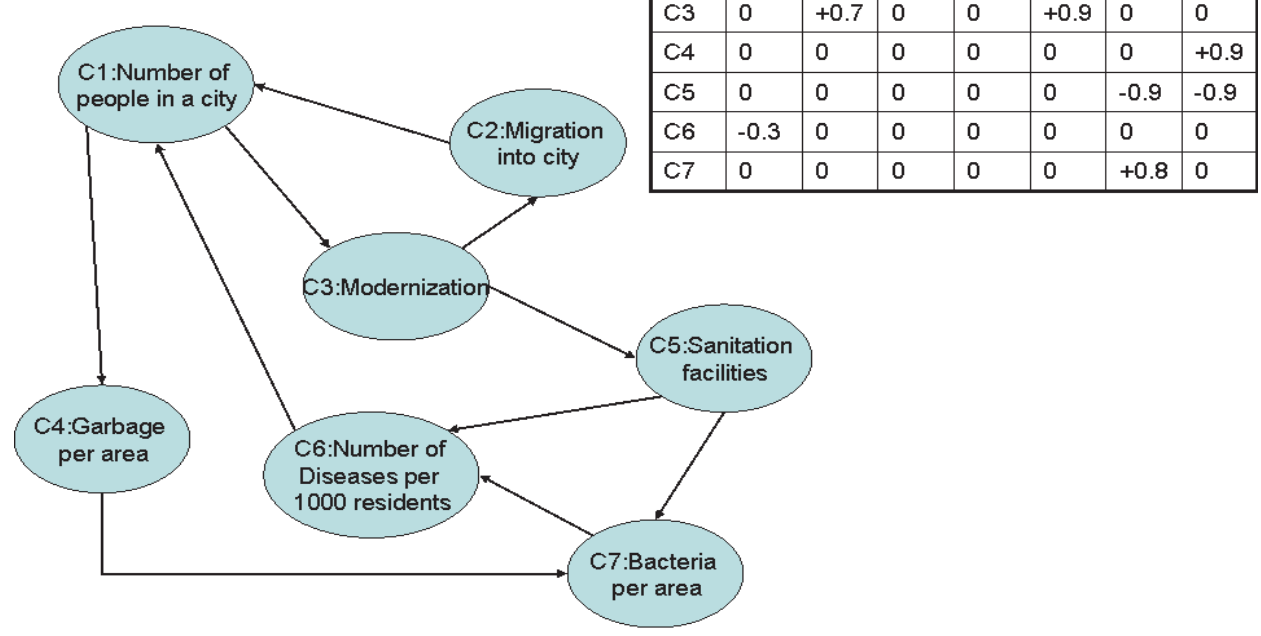

Fig. 1. Example of FCM model of the public health system: (a) FCM graph, and (b) connection matrix (adapted from Montazemi \& Conrath, 1986). 


\section{Data analysis using graph theory indices}

Graph theory methods help analyzing the structural properties of cognitive maps (Ozesmi \& Ozesmi, 2003). During the interviews, participants develop a FCM of the critical variables by drawing and circling the considerations they believe are important in relation to the topic under consideration. Then the main factors are defined and coded as concepts. The fuzzy directional arrows to one or more preceding factors are represented by fuzzy linguistic weights (see section 6), which after defuzzification produce a representative numerical weight. Using the defuzzification method of Centre of Gravity (COG) (Zadeh, 1976) a numerical weight is produced for each connection between concepts. CoG is computed from the following equation:

$$
\operatorname{COG}(B)=\frac{\sum_{q=1}^{N q} \mu_{B}\left(y_{q}\right) y_{q}}{\sum_{q=1}^{N q} \mu_{B}\left(y_{q}\right)}
$$

where $N q$ is the number of quantization used to discretize the membership function $\mu_{B}\left(y_{q}\right)$ of the fuzzy output $B$.

Thus they make-up a continuity map whereby concept $C_{i}$ is preceded by concept $C_{j}$ indicating a cause-and-effect relationship. Each individual map is analyzed in relation to the number of concepts, connections, connection-to-concept ratio, and density (calculated by dividing the number of connections in the map by the square value of concepts). To allow for identification of key criteria within the process of cognitive mapping, an analysis of domain and centrality is also conducted. The complexity level of each individual concept is revealed through a number of structural measures of cognitive maps, e.g. the centrality index borrowed from social networks analysis.

According to graph theory an effective way to better understand the structure of complex cognitive maps is condensing them. Condensation is achieved by replacing subgraphs (consisting of a group of variables connected with lines) with a single unit (Harary et al. 1965). Once the individual cognitive maps are drawn, they are qualitatively aggregated using clustering concepts to produce a condensed map named the collective FCM. Due to the complexity in FCM graphs (as the number of nodes and connections is often very large) the most central variables with their weighted connections are usually illustrated.

Analyzing the structure of cognitive maps is to look how connected or sparse the maps are. This is expressed by an index of connectivity, called density of a cognitive map (D). The density is equal to the number of connections divided by the maximum number of connections possible between $\mathrm{N}$ variables, thus $\mathrm{N}^{2}$. If the density of a map is high then the interviewee sees a large number of causal relationships among the variables.

The structure of a cognitive map apart from number of variables and connections can best be analyzed by finding transmitter variables (forcing functions, givens, tails, independent variables), receiver variables (utility variables, ends, heads, dependent variables) and ordinary variables (Bougon et al. 1977; Eden et al. 1992; Harary et al. 1965). These variables are defined by their outdegree and indegree. Outdegree is the row sum of absolute values of a variable in the adjacency matrix and shows the cumulative strengths of connections $\left(\mathrm{E}_{\mathrm{ij}}\right)$. It is a measure of 
how much a given variable influences other variables. Indegree is the column sum of absolute values of a variable and shows the cumulative strength of variables entering the unit.

Transmitter variables are units whose outdegree is positive and their indegree is 0 . Receiver variables are units whose outdegree is 0 and their indegree is positive. Other variables, which have both non-zero outdegree and indegree, are ordinary variables (means) (Eden et al., 1992, Ozesmi \& Ozesmi 2004). This type of variables reveals how people think about the causal relationships. For instance, if someone views a variable as a transmitter, this means that he perceives of the relative causal relationship as forcing function, which cannot be controlled by any other variables. In contrast, a receiver variable is seen as not affecting any of the other variables in the system. The total number of receiver variables in a cognitive map can be considered an index of its complexity. Larger number of receiver variables indicates that the cognitive map considers many outcomes and implications that are a result of the system (Eden, 1992). Many transmitter units show the "flatness" of a cognitive map where causal arguments are not well elaborated (Eden et al. 1992).

Centrality is the most important measure for map complexity, borrowed from social networks analysis, and is the summation of variable's indegree and outdegree (Bougon et al., 1977; Eden et al., 1992). Actually the centrality shows how connected the variable is to other variables and what the cumulative strength of these connections is. Another structural measure of cognitive maps is the hierarchy index (h), which is a function of the out-degrees and number of variables in a given map and represents the type of system as fully hierarchical, or democratic (see Ozesmi \& Ozesmi, 2004, pp. 50-51 for formulas).

\section{Development of Fuzzy Cognitive Maps}

The design of a fuzzy cognitive map is a process that heavily relies on the input from experts and/or stakeholders (Hobbs et al., 2002). This methodology extracts the knowledge from the stakeholders and exploits their experience of the system's model and behaviour. FCM is fairly simple and easy to understand for the participants, which opens up the possibility for involving lay people as well as planners, managers and experts (Isaac et al. 2009). Even though the cognitive nature of a FCM makes it inevitably a subjective representation of the system, Tan \& Özesmi (2006) emphasize that the model is not arbitrary as it is built carefully and reflexively with stakeholders (in groups or individually).

According to the FCM development process, at the first step of the construction process, the number and kind of concepts are determined by a group of experts and/or system stakeholders that comprise the FCM model. Then, a domain expert and/or stakeholder describe each interconnection either with an if-then rule that infers a fuzzy linguistic variable from a determined set or with a direct fuzzy linguistic weight, which associates the relationship between the two concepts and determines the grade of causality between the two concepts.

For example, someone can assign the strength of influence of concept $C_{j}$ on concept $C_{i}$ using the following form: "The strength of influence of concept $C_{j}$ on concept $C_{i}$ is T\{influence $\}$ " where the variable $T\{$ influence $\}$ declares the causal inter-relationships among concepts (i.e. the degree of influence from concept $C_{j}$ to $C_{i}$ ). Its term set $T\{$ influence\} is suggested to comprise thirteen variables and takes values in the universe $U=[-1,1]$. Using thirteen linguistic variables, an expert can describe in detail the influence of one concept on another and can discern between different degrees of influence. The thirteen variables used here are: T(influence) $=$ 
\{negatively very very strong, negatively very strong, negatively strong, negatively medium, negatively weak, negatively very weak, zero, positively very weak, positively weak, positively medium, positively strong, positively very strong, positively very very strong\}.

The corresponding membership functions for these terms are shown in Fig. 2 and they are $\mu_{n v v s,}, \mu_{n v s}, \mu_{n s}, \mu_{n m}, \mu_{n w}, \mu_{n v w}, \mu_{z}, \mu_{p v w}, \mu_{p w}, \mu_{p m}, \mu_{p s} \mu_{p v s}$ and $\mu_{p v v s .}$

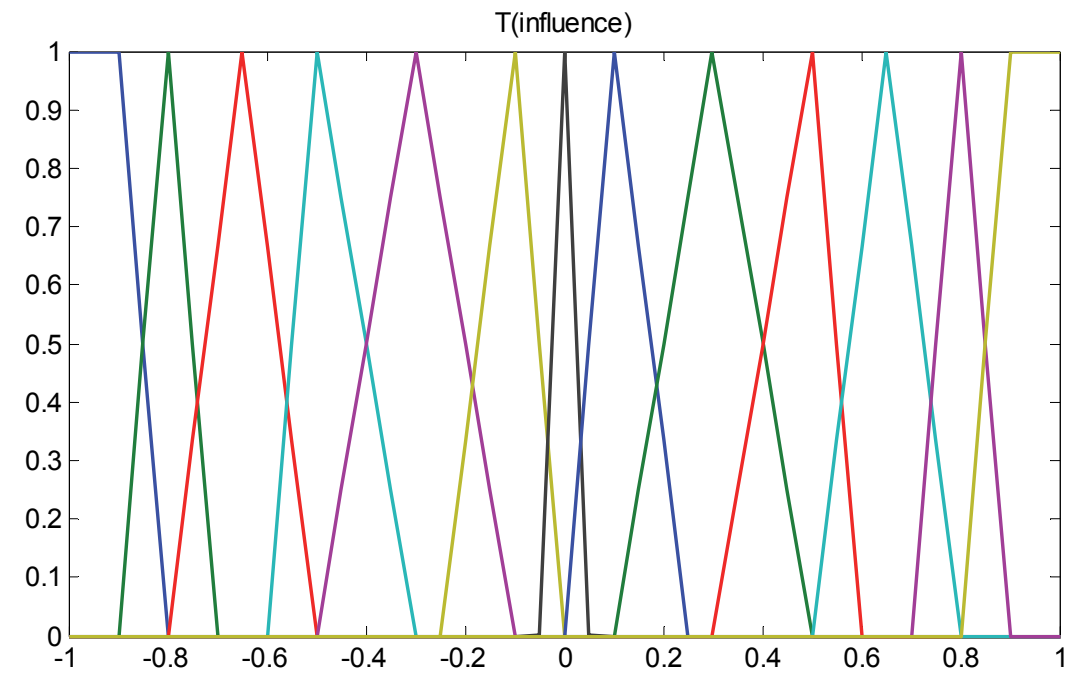

Fig. 2. The thirteen membership functions describing T(influence)

The inference of the rule $T\{$ influence $\}$ means that the linguistic weight $y\left(w_{i j}\right)$ is $\mu_{B}$, where $\mu_{B}$ is a linguistic variable from the set $T$. Also, experts and/or system stakeholders can directly assign the fuzzy linguistic weight $y$ (that describes the strength of connection between concepts $C_{i}$ and $C_{j}$ ) with no use of fuzzy rules. For example, someone can assign the strength of the connection between concepts $C_{i}$ and $C_{j}$ as follows: The influence from concept $C_{i}$ to concept $C_{j}$ is positively very high.

Finally, the linguistic variables $\boldsymbol{D}$ from the set T(influence) - proposed by the experts for each interconnection - are aggregated using the SUM method and so an overall linguistic weight is produced (Papageorgiou \& Stylios, 2008). Finally, the Center of Gravity (CoG) defuzzification method (Zadeh, 1986) is used for the transformation of the linguistic weight to a numerical value within the range $[-1,1]$. This methodology has the advantage that experts are not required to assign directly numerical values to causality relationships, but rather to describe qualitatively the degree of causality among the concepts. Thus, an initial matrix $W^{\text {initial }}=\left[\mathrm{W}_{\mathrm{ij}}\right]$, $\mathrm{i}, \mathrm{j}=1, \ldots, \mathrm{N}$, with $W_{\text {ii }}=0, \mathrm{i}=1, \ldots, N$, is obtained. Using the initial concept values, $A_{\mathrm{i}}$, which are also provided by the experts, the matrix $W$ initial is used for the determination of the steady state of the FCM, through the application of the rule of Eq. (2) or (5).

\section{The FCM inference and simulation processes}

Using artificial intelligent techniques, the dynamics of a fuzzy cognitive map can be traced analytically through a specific inference and simulation process. Each one of the $C_{j}$ concepts 
can take values in the unit interval $[0,1]$, also called the 'activation level'. The activation level can be interpreted as relative abundance (Hobbs et al. 2002). More rigorously, the activation level can represent membership in fuzzy set describing linguistic measures of relative abundance (e.g. low, average, high) (Kosko, 1986).

Values of the concept $C_{i}$ in time $t$ are represented by the state vector $A_{i}(t)$ while the state of the whole fuzzy cognitive map can be described by the state vector $\boldsymbol{A}(t)=\left[\boldsymbol{A}_{l}(t), \ldots, \boldsymbol{A}_{n}(t)\right]$ representing a point within a fuzzy hypercube that the system achieves at a certain point. The whole system with an input vector $\boldsymbol{A ( 0 )}$ describes a time trace within a multidimensional space $I^{n}$ that can gradually converge to an equilibrium point, or a chaotic point or a periodic attractor within a fuzzy hypercube. To which attractor the system will converge depends on the value of the input vector $\boldsymbol{A}(0)$.

The value $A_{i}$ of each concept $C_{i}$ in a moment $t+1$ is calculated by the sum of the previous value of $A_{i}$ in a precedent moment $t$ with the product of the value $\mathrm{A}_{j}$ of the cause node $C_{j}$ in precedent moment $t$ and the value of the cause-effect link $\mathrm{w}_{i j}$. The mathematical representation of the inference process of a fuzzy cognitive map has the following matrix form (Papageorgiou \& Stylios, 2008):

$$
\boldsymbol{A}^{(k)}=f\left(\boldsymbol{A}^{(k-1)}+\sum \boldsymbol{A}^{(k-1)} \cdot \boldsymbol{W}\right)
$$

Thus, the value $A_{j}$ for each concept $C_{j}$ is calculated by:

$$
A_{i}{ }^{(k+1)}=f\left(A_{i}^{(k)}+\sum_{\substack{j \neq i \\ j=1}}^{N} A_{j}^{(k)} \cdot w_{j i}\right)
$$

where $A_{i}^{(k+1)}$ is the value of concept $C_{i}$ at simulation step $k+1, A_{j}^{(k)}$ is the value of concept $C_{j}$ at step $k, w_{j i}$ is the weight of the interconnection between concept $C_{j}$ and concept $C_{i}$ and $f$ is a threshold (activation) function (Bueno \& Salmeron, 2008). Sigmoid threshold function gives values of concepts in the range $[0,1]$ and its mathematical type is:

$$
f(x)=\frac{1}{1+e^{-m x}}
$$

where $m$ is a real positive number and $x$ is the value $A_{i}^{(k)}$ on the equilibrium point. A concept is turned on or activated by making its vector element 1 or 0 in $(0,1)$. The sigmoid threshold function is used to reduce unbounded weighted sum to a certain range, which hinders quantitative analysis, but allows for qualitative comparisons between concepts (Bueno \& Salmeron, 2008).

A modified FCM inference algorithm, which updates the common FCM simulation process as initially suggested by Kosko (1986) can be used to avoid the conflicts that emerge in cases where the initial values of concepts are 0 or 0.5 , thus overcoming the limitation present by the sigmoid threshold function. This rescaled algorithm is implemented especially for the cases where there is no information about a certain concept/state or the expert/stakeholder 
cannot describe efficiently the initial state of a variable (Papageorgiou et al., 2010, Papageorgiou, 2011). Thus, the eq. (2) is transformed to the eq. (5).

$$
A_{i}(k+1)=f\left(\left(2 A_{i}(k)-1\right)+\sum_{\substack{j \neq i \\ j=1}}^{N}\left(2 A_{j}(k)-1\right) \cdot E_{j i}\right)
$$

The FCM simulation process is initialized through assigning a value between 0 and +1 to the activation level of each of the nodes of the map, based on experts/stakeholder opinion for the current state; then the concepts are free to interact. The value of zero suggests that a given concept is not present in the system at a particular iteration, whereas the value of one indicates that a given concept is present to its maximum degree. Other values correspond to intermediate levels of activation. The activation level of each concept depends on its value at the preceding iteration as well as on the preceding values of all concepts that exert influence on it through non-zero relationships. The simulation, which with regard to its content is mainly qualitative, is not intended to produce exact quantitative values. It aims at identifying the pattern of system's behaviour via the achieved values of the concepts of the FCM, which are progressively formed according to given considerations.

After defining all variables and necessary values, as well as the relationships between them, the simulation is carried out by use of the simulator consisting of the following five steps:

Step 1. Definition of the initial vector $A$ that corresponds to the concepts identified by suggestions and available knowledge.

Step 2. Multiply the initial vector $A$ and the matrix $W$ defined through equation (2) or (5)

Step 3. The resultant vector $A$ at time step $k$ is updating using eqs. (2) or (5) and (4).

Step 4. This new vector $\boldsymbol{A}^{k}$ is considered as an initial vector in the next iteration.

Step 5. Steps 2-4 are repeated until $\boldsymbol{A}^{k}-\boldsymbol{A}^{k-1} \leq e=0.001$ (where $e$ is a residual describing the minimum error difference among the subsequent concepts) or $\boldsymbol{A}^{k}=\boldsymbol{A}^{k-1}$. Thus $\boldsymbol{A} f$ $=\boldsymbol{A}^{k}$.

In each step of the cycling the values of concepts change according to the equation (2) or (5). This interaction between concepts continues until: i) a fixed equilibrium is reached, ii) a limited cycle is reached or iii) a chaotic behavior is exhibited. Actually, in most cases, the iteration stops when a limit vector is reached, i.e., when $\boldsymbol{A}^{k}=\boldsymbol{A}^{k-1}$ or when $\boldsymbol{A}^{k}-\boldsymbol{A}^{k-1} \leq e$; where $e$ is a residual, whose value depends on the application type (and in most applications is equal to 0.001 ). Thus, a final vector $A f$ is obtained.

In the previous analysis, all type of information has numerical values. FCM allows us to perform qualitative simulations and experiment with a dynamic model. Simulations allow for analysis of several aspects of FCMs, such as concepts activation levels at the final state (if there are any) and changes/trends in the activation levels throughout the simulation concerning either all concepts or a subset of concepts that is of interest to the user, and discovery of cycles (intervals, concepts activation levels within the cycle). This type of analysis allows investigating "what-if" scenarios by performing simulations of a given model from different initial state vectors. Once an FCM has been subjected to an initial stimulus, it is possible to gain insight into a system's behaviour by studying the resulting stable state or cycle of states. Simulations offer description of dynamic behaviour of the system that can be used to support decision-making or predictions about its future states (Stach et al., 2010). 


\section{Steps in designing and implementing fuzzy cognitive mapping}

As with many other interview techniques, it is helpful to produce systematic guidelines describing the single steps of FCM before starting with the interviewing. These interview guidelines should function as a guidance/inspiration for how to conduct the interviews, and how to create FCMs over the case study areas. In this section we summarize the practical steps needed to design and conduct a FCM exercise. At first, how to draw a FCM must be explained to the interviewee(s)/stakeholder(s) using a cognitive map and its related FCM as an example. Once the interviewees understood the process of constructing a fuzzy cognitive map, then they are able to draw their own map of the issue under investigation following the steps below:

Step 1: Identification of factors.

Based on the guidelines, each one of the interviewee is asked to identify the main factors which come to his/her mind when he/she is asked about the topic been investigated, e.g. the future environmental risks in the Black Sea, seen as a system where humans, marine animals and plants are all living together. After the identification of the main factors affecting the environmental topic under investigation, each stakeholder is asked to describe the existence and type of the causal relationships among these factors and then assesses the strength of these causal relationships using a predetermined scale, capable to describe any kind of relationship between two factors, positive and negative. Thus, a FCM from each interviewee is established presenting the main factors/variables and the relationships among them and illustrating the individual's perceptions about the topic under investigation.

Step 2: Clustering of individual issues in more general concepts.

After the individual perceptions are elicited by interviews, a number of individual maps are produced. It is essential that the original concepts-variables, as described by lay people from interview, be clustered in more generic or more specific concepts, because most of them present the same meaning with a different word. Using experts' judgement, the importance of the original factors is discussed and they are then clustered. This can also be done through the construction of an ontological tree. This process of condensation enables aggregation of variables into high-level concepts, which then feed into the construction of the collective FCM.

Step 3: Estimation of causal link strengths in collective FCMs

The individual maps are then turned into a representative, collective map. To achieve this, all the suggested strength relations by lay people are transferred into linguistic variables using the aggregation method of SUM to obtain an overall linguistic weight. Following this, defuzzification turns linguistic weights into numerical weights in the range of $[-1,1]$. This condensed map is analyzed using the established indicators of out-degree, in-degree, centrality, density, hierarchy as well as the transmitter, receiver, and ordinary variables. Through this analysis the collective map is explained demonstrating its usefulness for identifying policies vis-à-vis individual FCMs.

Step 4: FCM simulations

Next a number of simulations are performed using the inference process given by equations (2) and (5). The calculated output of the FCM model shows how the system reacts under the assumptions given by the stakeholders or related users. Usually, the calculated output is different from the expected one, thus presenting a potential added value of Fuzzy Cognitive Map as a decision support tool. 


\section{An application of FCM in the Black Sea}

In order to illustrate in brief the methods described so far, we present a FCM exercise designed and implemented in the Northern Black Sea with Ukrainian stakeholders (for details see Kontogianni et al., forthcoming). In this application we were interested in investigating how the citizens perceive the future prospects and risks of the Black Sea marine environment; creating and analyzing their FCMs this can be achieved. We employed 29 in-depth lay people interviews (see Appendix A). Based on specific guidelines, each interviewee was asked to identify at first the main factors which come to his/her mind when he/she is asked about the Black Sea as a system where humans, marine animals and plants are all living together. During the interviews participants developed thus a FCM of the critical variables (important considerations) by drawing and circling the considerations they believe are important for environmental health of marine state ecosystem in the Black Sea area.

After the identification of the main factors affecting the environmental health of the marine ecosystem in the Black Sea, each stakeholder was asked to describe the existence and type of the causal relationships among these factors and then, the strength of the causal relationships-influences that may exist between these factors. This phase was implemented 13 grades scale, numbering from -6 to +6 , capable to describe any kind of relationship between two factors, positive and negative (see Table 1).

Thus, a FCM from each interviewee was established presenting the main factors/variables and the relationships among them illustrating the individual's perceptions about the future prospects and the risks about the ecological health of the marine environment in the Black Sea. Figure 3 illustrates the produced FCM defined by an individual/stakeholder from Ukraine for further assessment.

The initial number of important factors identified by stakeholders was 52 . Since it was decided to produce a collective FCM providing detail for future risks-related issues we limited the number of factors having the same meaning through clustering. Using marine experts' judgement, the importance of the original 52 factors was discussed and then clustered in a total of 26 concepts (Table A in Appendix). The mean number of variables in the individual cognitive maps of the Black Sea ecosystem drawn by the 29 respondents was $7.86 \pm 1.7$, with $11 \pm 6.513$ connections on average between the variables that they defined. There were a total of 26 variables with 145 connections in the collective cognitive map obtained by clustering and augmented the 29 individual FCMs.

The process of condensation enabled aggregation of variables into high-level concepts, which then feed into the construction of a collective FCM for Ukrainian stakeholders. The collective FCM (consisting of 26 concepts and 145 relationships among concepts) is thus obtained (see Figure 4 developed in pajek software [http://vlado.fmf.unilj.si/pub/networks/pajek/]).

The collective FCM was then coded as adjacency matrix $E=\left[\mathrm{e}_{i j}\right]$ and its structure was analyzed using the indices derived from graph theory (see section 4). Due to the complexity of the collective FCM graph (as the number of nodes and connections is very large) Figure 5 illustrates the most central variables with their weighted connections.

It is observed from graph indices calculations that the density of collective FCM is high and a mentioned complexity is present. A relatively high complexity is considered in the cases where the receiver variables are more than the transmitter variables, and in our case, the complexity is equal to 1.5 (complexity $>1$ means relatively high complexity). The most 
frequently mentioned ( $>3$ times) variables that were recurrent in the 29 fuzzy cognitive maps are: 'Coastal Development', 'Biodiversity', 'Tourism', and 'Municipal Solid Waste'.

\begin{tabular}{|l|l|l|}
\hline $\begin{array}{l}\text { Strength connection by lay } \\
\text { people }\end{array}$ & $\begin{array}{l}\text { Sign and Strength of relationship } \\
\text { (Linguistic weight) }\end{array}$ & $\begin{array}{l}\text { Interpreted crisp } \\
\text { weight }\end{array}$ \\
\hline-6 & Negatively very very strong & -1 \\
\hline-5 & Negatively very strong & -0.9 \\
\hline-4 & Negatively strong & -0.75 \\
\hline-3 & Negatively medium & -0.5 \\
\hline-2 & Negatively weak & -0.3 \\
\hline-1 & Negatively very weak & -0.1 \\
\hline 0 & Zero & 0 \\
\hline 1 & Positively very weak & 0.1 \\
\hline 2 & Positively weak & 0.3 \\
\hline 3 & Positively medium & 0.5 \\
\hline 4 & Positively strong & 0.75 \\
\hline 5 & Positively very strong & 0.9 \\
\hline 6 & Positively very very strong & 1 \\
\hline
\end{tabular}

Table 1. Interpretation of lay people's strength connections among concepts to crisp weights in the range $[-1,1]$.

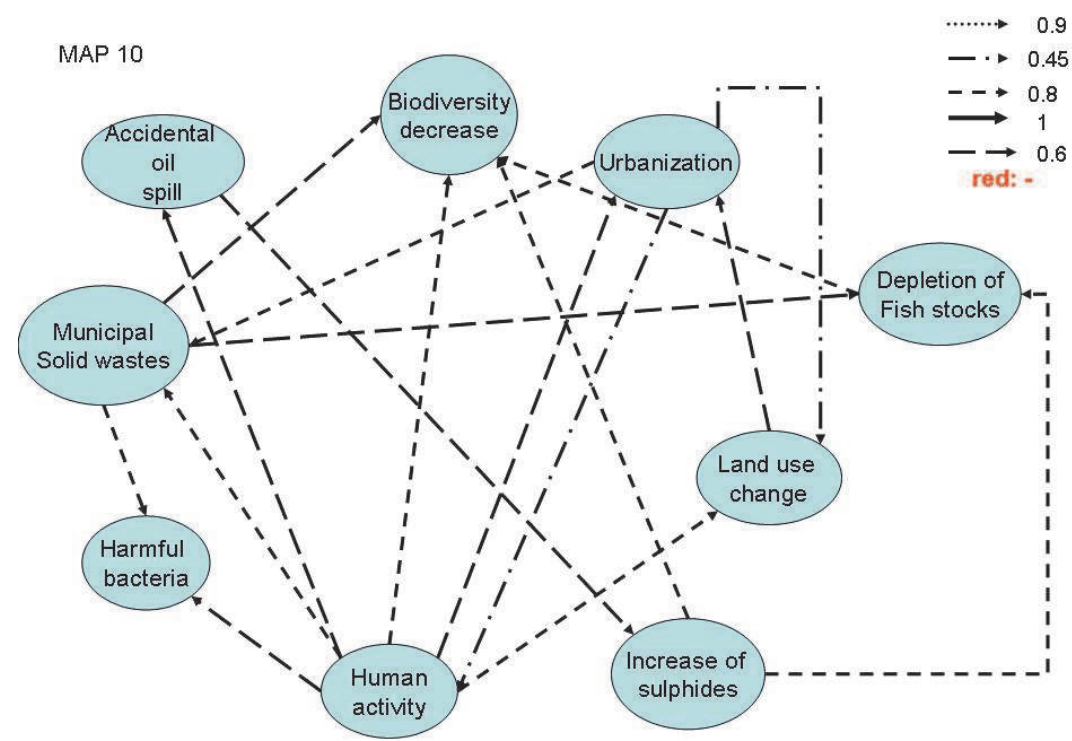

Fig. 3. The individual FCM defined by an individual/stakeholder from Ukraine. 


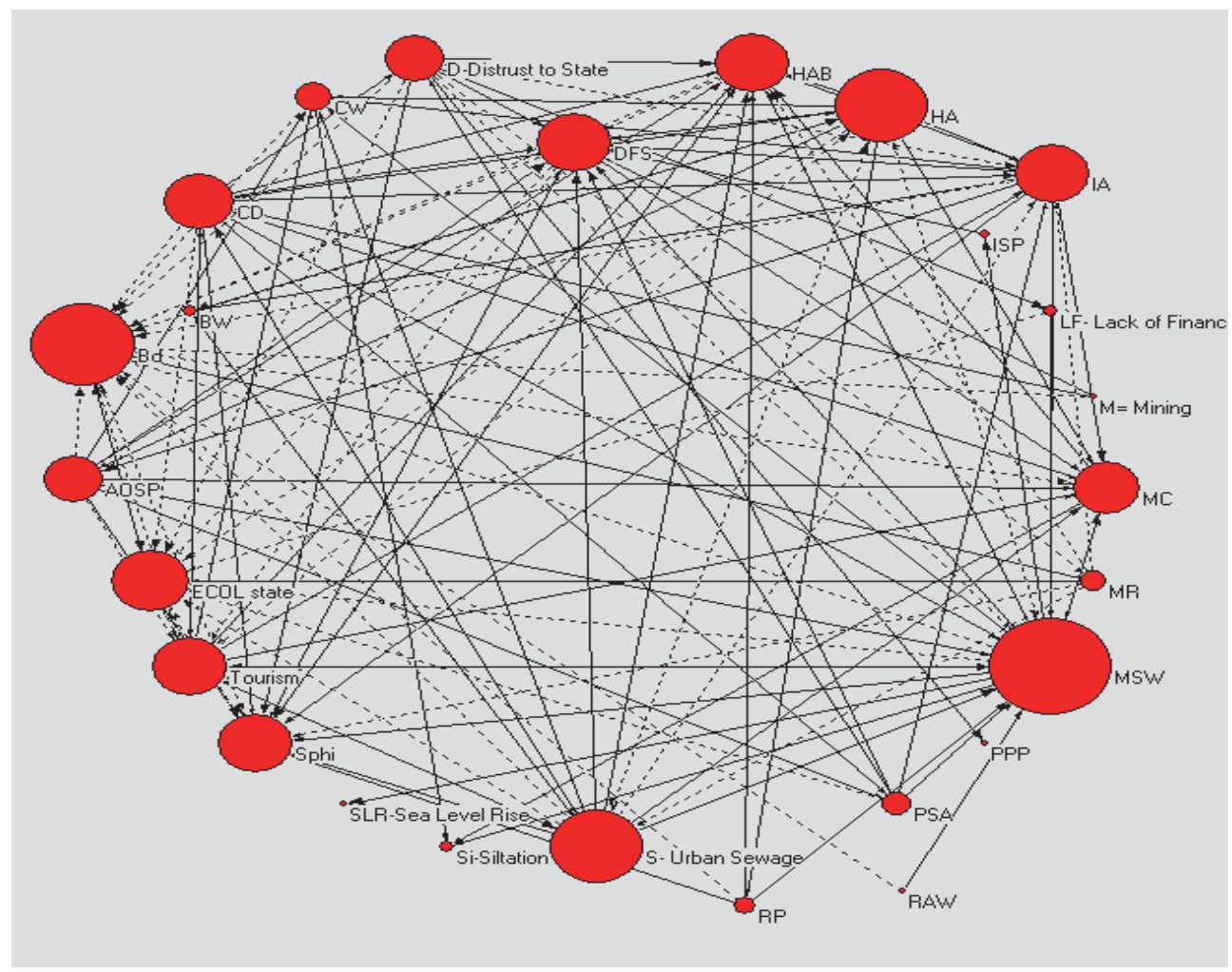

Fig. 4. The collective fuzzy cognitive map of Ukrainian stakeholders. 


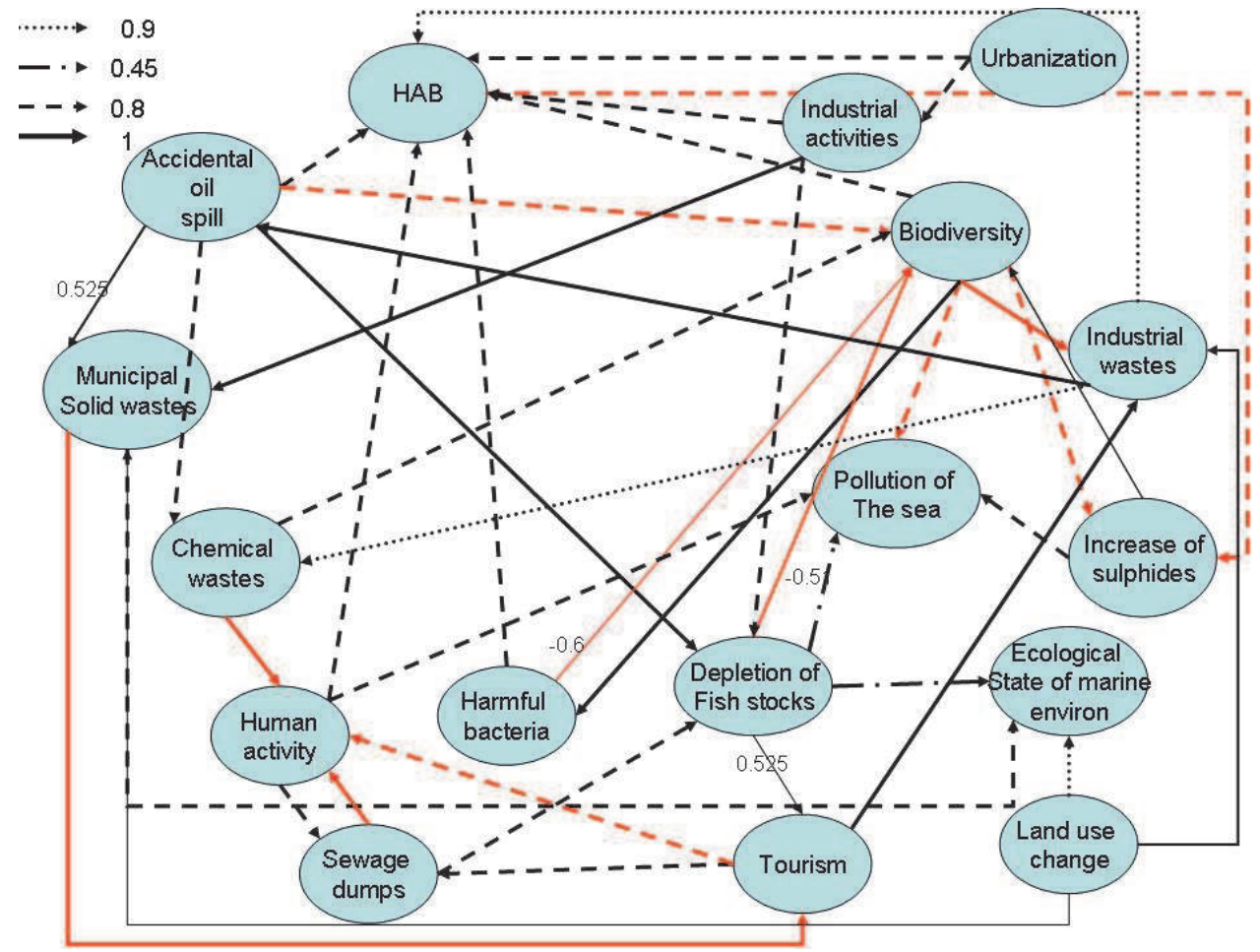

Fig. 5. The collective FCM with most central variables and strong connections.

Table 2 gathers the calculated graph theory indices for the collective map and the sum of the 29 individual maps.

\begin{tabular}{|l|l|l|}
\hline Indices & Individual Maps & Collective FCM \\
\hline Maps & 29 & 1 \\
\hline Variables (N) & $7.86 \pm 1.574$ & 26 \\
\hline Number of connections (W) & $11 \pm 6.513$ & 145 \\
\hline No. of transmitter variables (T) & $2.21 \pm 1.544$ & 2 \\
\hline No. of receiver variables (R) & $1.96 \pm 1.267$ & 3 \\
\hline No. of ordinary variables (O) & $3.21 \pm 1.445$ & 21 \\
\hline Connection/Variable (W/N) & $1.33 \pm 0.617$ & 4.577778 \\
\hline Complexity (Receiver/Transmitter) & $1.02 \pm 1.198$ & 1.5 \\
\hline Density (D=W/N^2) & $0.167 \pm 0.065$ & 0.101728 \\
\hline Hierarchy index (h) & $0.017 \pm 0.005$ & 0.012944 \\
\hline
\end{tabular}

Table 2. Average $( \pm \mathrm{SD})$ graph theoretical indices of the individual FCMs and the indices of the collective FCM.

The collective FCM was then used for analyzing system behaviour and to run management simulations. Simulations were generated using both inference equations, eq. (2) and rescaled eq. (5), by taking the product of the vector of initial states of variables $\left(\mathbf{A}_{\mathbf{0}}\right)$ times the square 
matrix $E$ of the collective map. Simulations were generated using the five steps described previously implemented in Matlab R2008a environment for Windows. At first, we determine the steady state condition of the system's convergence before we consider any management options. We are doing this in order to be able to see the perceived tendency of the Black Sea ecosystem based on the collective cognitive map and test for its internal coherence. For this purpose, we run the generic FCM model with 50 different random initial states for all variables between 0 and 1 drawn from a uniform distribution. In all of these nomanagement simulations, the system reached a steady state after 25-30 iterations, where some of the produced final steady states for each initial condition were different. By excluding a chaotic behavior, we accordingly confirm a dynamically stable and coherent mental structure of our sample.

Then, we consider a number of assumptions for the concepts and risk factors, which affect the future state of the environmental health of marine ecosystem in the Black Sea to analyze the system performance and its decision-making capabilities. Initially, we considered a case where all concepts are set to zero. This means that all concepts are not activated for this specific consideration. After 23 iterations the FCM system reaches a final state where the concepts "Biodiversity" and "Ecological State" exhibit very high values and therefore increase the environmental health of marine ecosystem. This is an upper bound for the Black Sea ecosystem health, conditioned by the basic structure of respondents' cognitive reality.

In a next step, we consider a case where all concepts are set to one, meaning that the 24 factors are fully activated under this assumption. After 20 iterations the system reaches a final state where "Biodiversity" and "Ecological State" respectively clamp to zero. This is a lower bound for the Black Sea ecosystem health, conditioned by the basic structure of respondents' cognitive reality.

In-between these two extreme cases, we develop a number of policy scenarios, based on a number of ad hoc interventions for the Black Sea ecosystem conservation. In these interventions, individual concepts and groups of concepts consequently were considered to be activated and the final state of FCM system under these scenarios determined. Each simulation runs under two different versions where the activated concepts are set either one or 0.5. The rationale for this approach is to test the influence of uncertainty in the functioning of the other concepts. The value of 0.5 means there is uncertainty concerning the true state of the impact of the other concepts. The calculated output of the FCM model shows how the system reacts under the assumptions given by the stakeholders or related users. Usually, the calculated output is different from the expected one, thus presenting a potential added-value of Fuzzy Cognitive Map as a policy making tool.

One sample policy making scenario is presented. The five most central concepts- MSW (Municipal Solid Wastes), HA (Human Activities), Urban Sewage, IA (Industrial Activities), HAB (Harmful Algal Blooms)- are considered as the only de-activated concepts whereas all the other 21 concepts are considered as activated concepts that take values: (a) equal to 1 and (b) equal to 0.5 , depicting strong activation or uncertainty state. Table 3 depicts the calculated values of all concepts in the final state for the considered scenarios (a) and (b), main observations being the high values of Biodiversity $(\mathrm{Bd})$ and Ecological State of marine environment (ECOL). A significant increase to Biodiversity and ECOL state is a potential outcome of conservation policies regulating the input of those five most central concepts acting as risk factors. Thus the future state of the marine ecosystem could be improved if the five most central concepts might decrease at a significant amount. 


\begin{tabular}{|c|c|c|c|c|c|c|}
\hline Concepts & $\begin{array}{c}\text { Initial } \\
\text { values- } \\
\text { Scenario } \\
\text { (a) }\end{array}$ & $\begin{array}{l}\text { Final state } \\
\text {-eq. (5) } \\
\text { Scenario } \\
\text { (a) }\end{array}$ & $\begin{array}{l}\text { Final state } \\
\text {-eq. (2) } \\
\text { Scenario } \\
\text { (a) }\end{array}$ & $\begin{array}{c}\text { Initial } \\
\text { values- } \\
\text { Scenario } \\
\text { (b) }\end{array}$ & $\begin{array}{l}\text { Final state - } \\
\text { eq. (2) } \\
\text { Scenario } \\
\text { (b) }\end{array}$ & $\begin{array}{l}\text { Final state- } \\
\text { eq. (5) } \\
\text { Scenario } \\
\text { (b) }\end{array}$ \\
\hline AOSP & 1.00 & 0.7103 & 0.8808 & 0.5 & 0.8800 & 0.2835 \\
\hline Bd & 1.00 & 0.0004 & 0.0005 & 0.5 & 0.0007 & 0.9974 \\
\hline BW & 1.00 & 0.7290 & 0.8069 & 0.5 & 0.8063 & 0.3024 \\
\hline $\mathrm{CD}$ & 1.00 & 0.8113 & 0.9097 & 0.5 & 0.8868 & 0.3855 \\
\hline $\mathrm{CW}$ & 1.00 & 0.8558 & 0.9721 & 0.5 & 0.9718 & 0.1635 \\
\hline D-Distrust to State & 1.00 & 0.8284 & 0.9303 & 0.5 & 0.9295 & 0.3751 \\
\hline DFS & 1.00 & 0.9903 & 0.9974 & 0.5 & 0.9972 & 0.0174 \\
\hline HAB & 0 & 0.9938 & 0.9986 & 0 & 0.9986 & 0.0162 \\
\hline HA & 0 & 0.7297 & 0.3376 & 0 & 0.3346 & 0.2689 \\
\hline IA & 0 & 0.5125 & 0.7095 & 0 & 0.7049 & 0.4638 \\
\hline ISP & 1.00 & 0.7903 & 0.8367 & 0.5 & 0.8365 & 0.2359 \\
\hline LF & 1.00 & 0.7103 & 0.8162 & 0.5 & 0.8161 & 0.4114 \\
\hline M- Mining & 1.00 & 1.0000 & 1.0000 & 0.5 & 0.5000 & 0.5000 \\
\hline $\mathrm{MC}$ & 1.00 & 0.9842 & 0.9924 & 0.5 & 0.9922 & 0.0235 \\
\hline MR & 1.00 & 0.3199 & 0.4806 & 0.5 & 0.4808 & 0.5738 \\
\hline MSW & 0 & 0.9664 & 0.9964 & 0 & 0.9950 & 0.0955 \\
\hline PPP & 1.00 & 0.7270 & 0.8281 & 0.5 & 0.8280 & 0.4026 \\
\hline PSA & 1.00 & 0.7581 & 0.8754 & 0.5 & 0.8737 & 0.3187 \\
\hline RAW & 1.00 & 1.0000 & 1.0000 & 0.5 & 0.5000 & 0.5000 \\
\hline $\mathrm{RP}$ & 1.00 & 0.6315 & 0.7144 & 0.5 & 0.7140 & 0.3678 \\
\hline S- Urban Sewage & 0 & 0.7332 & 0.8591 & 0 & 0.8572 & 0.3698 \\
\hline Si-Siltation & 1.00 & 0.9169 & 0.9379 & 0.5 & 0.9378 & 0.0933 \\
\hline SLR & 1.00 & 0.8314 & 0.8655 & 0.5 & 0.8653 & 0.1948 \\
\hline Sphi & 1.00 & 0.9970 & 0.9953 & 0.5 & 0.9952 & 0.0061 \\
\hline Tourism & 1.00 & 0.2055 & 0.1535 & 0.5 & 0.1520 & 0.7777 \\
\hline ECOL & 1.00 & 0.0026 & 0.0055 & 0.5 & 0.0056 & 0.9913 \\
\hline
\end{tabular}

Table 3. Initial and final concepts' state after 25 iterations for Scenario (a) and (b). 


\section{Summary and conclusions}

In this chapter, the FCM methodology was presented and analyzed for the elicitation and understanding of individual and collective knowledge, preferences and beliefs. The aim was to present to the reader both a theoretical underpinning of FCMs as well as a grasp of their empirical modalities.

A cognition model, like FCM, represents a system in a form that corresponds closely to the way humans perceive it. Therefore, the model is easily understandable, even by a nonprofessional audience and each parameter has a perceivable meaning. The model can be easily altered to incorporate new phenomena, and if its behavior is different than expected, it is usually easy to find which factor should be modified and how. In this sense, a FCM is a dynamic modeling tool in which the resolution of the system representation can be increased by applying a further mapping. The FCM methodology developed makes it possible, if the initial mapping of the risk factors and future prospects of marine ecosystem is incomplete or incorrect, to make further additions to the map, and to predict the effects of the new parameters considered.

FCMs have some specific advantageous characteristics over traditional mapping methods: they capture more information in the relationships between concepts, are dynamic, combinable, and tunable, and express hidden relationships (Kosko, 1986, 1992). The resulting fuzzy model can be used to analyze, simulate, and test the influence of parameters and predict the behavior of the system. Summarizing, FCM helps describe the schematic structure, represent the causal relationships among the elements of a given decision environment, and the inference can be computed by a numeric matrix operation. With FCM it is usually easy to find which factor should be modified and in which way.

To illustrate the FCM methodology, an empirical application for modelling lay people perceptions is presented. We describe the main features of a FCM exercise designed to elicit the Black Sea stakeholder views/ perceptions about the risks that the Black Sea may face in the future 20 years. A generic model for environmental management is constructed by augmenting the individual FCMs drawn by lay people-stakeholders from Ukraine. The graph theoretical indices were calculated out of the individual cognitive maps and the collective cognitive map produced by augmentation. A number of scenarios were run using the FCM inference process to enable us to understand the complex structure of the Black Sea problems and the risks mainly affecting its marine ecosystem. This knowledge is further used to design policies that contribute in environmental management. The results show its functionality and demonstrate that the use of FCMs is reliable and efficient for this task.

\section{Acknowledgment}

This research was carried out within the IP project SESAME funded by the EU under Framework Programme 6, Key Action "Global change, climate and biodiversity", Contract No. 2006-036949. We thank the Coordinator Dr. E. Papathanassiou for his encouragement to apply early in 2008 Fuzzy Cognitive Mapping in marine governance.

\section{Appendix A}

Interview protocol

Stage (1): A Formal Introduction 
Which is the Aim of the study?

What is cognitive mapping methodology? Example cognitive map and fuzzy cognitive map from the public health study is depicted. Explanation on main factors and how these factors are interrelated is presented.

Stage (2) Creation of individual FCM

Which factors, which things, come into your mind spontaneously if I mention to you the Mediterranean (Black) Sea as a system where humans, marine animals and plants are all living together?

Is there any positive or negative relationship between these factors?

How strongly a factor A influences another factor B? A scale having 12 grades capable to describe any kind of relationship between two things is given.

Stage (3): Conclusion

Strong words/phrases they used, general comments of the interview.

\section{Appendix B}

\begin{tabular}{|c|c|c|c|c|c|}
\hline Concepts & Abbreviation & $\begin{array}{l}\text { Description } \\
\text { of concepts }\end{array}$ & Concepts & Abbreviation & $\begin{array}{l}\text { Description } \\
\text { of concepts }\end{array}$ \\
\hline $\mathrm{C} 1$ & AOSP & $\begin{array}{l}\text { Accidental } \\
\text { oil spill } \\
\text { pollution }\end{array}$ & $\mathrm{C} 14$ & Sphi & $\begin{array}{l}\text { Sulphide } \\
\text { Increase }\end{array}$ \\
\hline $\mathrm{C} 2$ & Tourism & Tourism & C15 & IA & $\begin{array}{l}\text { Industrial } \\
\text { Activities or } \\
\text { Industrial } \\
\text { Pollution }\end{array}$ \\
\hline $\mathrm{C} 3$ & PST & $\begin{array}{l}\text { Pollution of } \\
\text { Sea Trade }\end{array}$ & C16 & $S$ & $\begin{array}{l}\text { Urban } \\
\text { Sewage }\end{array}$ \\
\hline $\mathrm{C} 4$ & CD & $\begin{array}{l}\text { Coastal } \\
\text { Development }\end{array}$ & $\mathrm{C} 17$ & ISP & $\begin{array}{l}\text { Invasive } \\
\text { species }\end{array}$ \\
\hline C5 & BD & Biodiversity & C18 & MSW & $\begin{array}{l}\text { Municipal } \\
\text { solid waste } \\
\text { pollution to } \\
\text { the sea }\end{array}$ \\
\hline C6 & ChemW & $\begin{array}{l}\text { Chemical } \\
\text { wastes }\end{array}$ & C19 & MR & $\begin{array}{l}\text { MR= Marine } \\
\text { Research }\end{array}$ \\
\hline $\mathrm{C} 7$ & D & $\begin{array}{l}\text { Distrust to } \\
\text { State \& } \\
\text { Institutions }\end{array}$ & $\mathrm{C} 20$ & $\mathbf{R P}$ & $\begin{array}{l}\text { Riverine } \\
\text { Pollutants }\end{array}$ \\
\hline $\mathrm{C} 8$ & DFS & $\begin{array}{l}\text { Depletion of } \\
\text { Fish Stocks }\end{array}$ & $\mathrm{C} 21$ & SLR & Sea level rise \\
\hline C9 & RAW & Radio-active & $\mathrm{C} 22$ & BW & Ballast \\
\hline
\end{tabular}




\begin{tabular}{|c|c|c|c|c|c|}
\hline & & Waste & & & $\begin{array}{l}\text { Waters (ship } \\
\text { dumps) }\end{array}$ \\
\hline C10 & PPP & $\begin{array}{l}\text { Polluter } \\
\text { Pays } \\
\text { Principle }\end{array}$ & C23 & Si & Siltation \\
\hline C11 & LF & $\begin{array}{l}\text { Luck of } \\
\text { Financing }\end{array}$ & C24 & $\mathbf{M}$ & Mining \\
\hline C12 & MP & $\begin{array}{l}\text { Microbio- } \\
\text { logical } \\
\text { Pollution }\end{array}$ & C25 & HAB & $\begin{array}{l}\text { Harmful } \\
\text { Algae } \\
\text { Blooms }\end{array}$ \\
\hline $\mathrm{C} 13$ & HA & $\begin{array}{l}\text { Human } \\
\text { activity }\end{array}$ & C26 & ECOL & $\begin{array}{l}\text { Ecological } \\
\text { State of the } \\
\text { marine } \\
\text { environment }\end{array}$ \\
\hline
\end{tabular}

Table A. 26 clustered concepts describing stakeholders' perceptions.

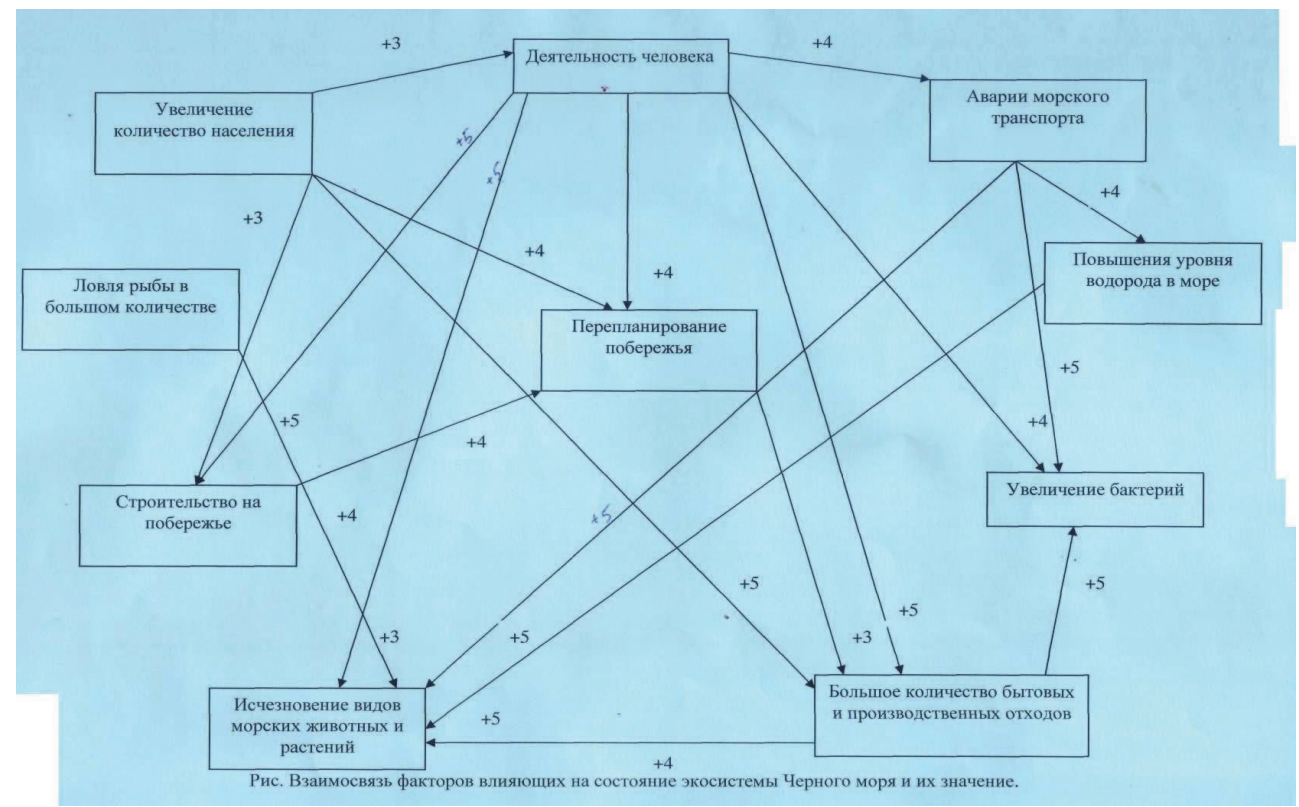

Fig. B1. A cognitive map defined by an individual/stakeholder from Ukraine. 


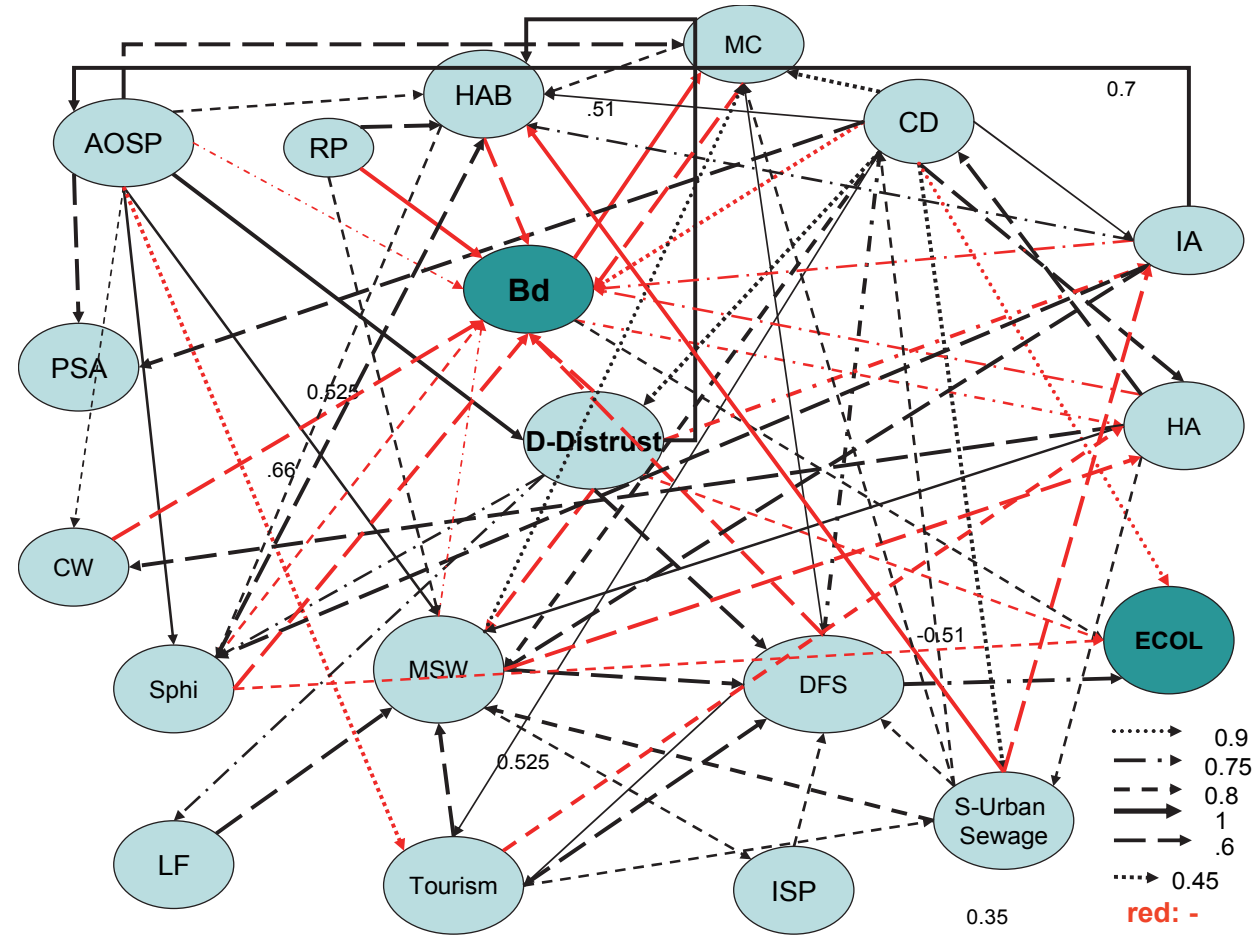

Fig. B2. The collective FCM with the eighteen most mentioned concepts and their related interconnections.

\section{References}

Biloslavo, R.; \& Dolinšek, S. (2010). Scenario planning for climate strategies development by integrating group Delphi, AHP and dynamic fuzzy cognitive maps. Foresight, vol. 12, no.2, pp. 38-48.

Brouwer, R.; Powe, N.; Turner, R.K.; Bateman, I.J. \& Langford, I.H. (1999). Public Attitudes to Contingent Valuation and Public Consultation. Environmental Values vol. 8 no. 3, pp. 325-347

Bougon, M., Weick, K., and Binkhorst, D. (1977). Cognition in organizations: An analysis of the Utrecht jazz orchestra. Administrative Science Quarterly, vol. 22, pp.606-639.

Bueno S., \& J.L. Salmeron (2008). Fuzzy modeling Enterprise Resource Planning tool selection. Computer Standards \& Interfaces, vol. 30 137-147.

Buzan T. (1993). The Mind Map Book. London: BBC Books

Carley, K. \& M. Palmquist, 1992. Extracting, representing, and analyzing mental models. Social Forces vol. 70, pp.601-636.

Contreras J., Juan P. Paz, David Amaya \& Pineda, A. (2007). Realistic Ecosystem Modelling with Fuzzy Cognitive Maps. International Journal of Computational Intelligence Research. ISSN 0973-1873 Vol.3, No.2, pp. 139-144 
Craiger, J. P. \& Coovert, M. D. (1994). Modeling dynamic social and psychological processes with fuzzy cognitive maps. Proceedings of the Third IEEE World Conference on Fuzzy Systems, vol. 3, pp. 1873-1877.

Desvousges W. H. and V. K. Smith (1988). Focus Groups and Risk Communication: The "Science" of Listening to Data. Risk Analysis vol. 8, no. 4, pp.479-484

Eden C. (1992). On the nature of cognitive maps. Journal of Management Studies vol. 29, pp. 261-265.

Eden C; Ackermann F, \& Cropper S (1992). The analysis of cause maps. Journal of Management Studies vol. 29, pp. 309-324.

Eisenack K.; M.K.B. Lüdeke; G. Petschel-Held, J. Scheffran \& Kropp, J.P. (2006). Qualitative modeling techniques to assess patterns of global change. In: J.P. Kropp and J. Scheffran, Editors, Advanced Methods for Decision Making and Risk Management in Sustainability Science, Nova Science Publishers, Hauppage, NY, USA.

Grimble R. \& Wellard, K. (1997). Stakeholder Methodologies in Natural Resource Management: a Review of Principles, Contexts, Experiences and Opportunities. Agricultural Systems, vol. 55, No. 2, pp. 173-193.

Grosskurth J. \& Rotmans, J. (2005). The scene model: getting a grip on sustainable development in policy making, Environment development and sustainability vol. 7, no. 1, pp. 135-151.

Grosskurth J. (2008). Regional Sustainability: tools for integrative governance, Maastricht University, Maastricht.

Harary, F.; Norman R. Z. \& D. Cartwright, (1965). Structural Models: An Introduction to the Theory of Directed Graphs. John Wiley \& Sons, New York.

Hermans, L. M. (2008). Exploring the promise of actor analysis for environmental policy analysis: lessons from four cases in water resources management. Ecology and Society, vol.13, no. 1, pp. 21.

[online URL: http://www. ecologyandsociety.org/vol13/iss1/art21/]

Hjortsø CN; Christensen SM; \& Tarp P (2005). Rapid stakeholder and conflict assessment for natural resource management using cognitive mapping: the case of Damdoi Forest Enterprise, Vietnam. Agriculture and Human Values vol. 22, pp. 149-167.

Hobbs, B. F., S. A. Ludsin, R. L. Knight, P. A. Ryan, J. Biberhofer \& Ciborowski, J. J. H. (2002). Fuzzy cognitive mapping as a tool to define management objectives for complex ecosystems. Ecological Applications vol. 12, pp.1548-1565.

Isaac M. E., Dawoe, E. \& Sieciechowicz, K. (2009). Assessing Local Knowledge Use in Agroforestry Management with Cognitive Maps. Environmental Management vol. 43, pp. 1321-1329.

Karageorgis A., Kapsimalis V. Kontogianni A., Skourtos M. Turner R.K, Salomons W (2006) Impact of 100-year human interventions on the Deltaik coastal zone of the inner Thermaikos Gulf (Greece): A DPSIR framework analysis. Journal of Environmental Management 38, 304-315

Kardaras, D., \& Karakostas, B. (1999). The use of fuzzy cognitive maps to simulate the information systems strategic planning process. Information and Software Technology, vol. 41, pp.197-210. 
Khan, M.S., Quaddus, M.A., \& Intrapairot, A. (2001). Application of a Fuzzy Cognitive Map for Analysing Data Warehouse Diffusion. Applied Informatics: Artificial Intelligence and Applications; Advances in Computer Applications. Proceedings of IASTED International Symposia. 19-22 February, Innsbruck, Austria, pp.32-37.

Kontogianni A., Skourtos M., Langford I., Bateman I., Georgiou S. (2001), Integrating Stakeholder Analysis in non- market valuation of environmental assets, Ecological Economics 37: 123-138, Elsevier Publishers

Kontogianni A., Tziritis I., Skourtos M. (2005), Bottom-up environmental decision making taken seriously: Integrating stakeholder perceptions into scenarios of environmental change. Human Ecology Review 12.2, 87-95

Kontogianni A., M. Skourtos (2008), Social Perception of Risk informing Integrated Coastal Zone Management on accidental oil - spill pollution: 'the reason you pollute matters, not numbers', in book: "Integrated Coastal Zone Management - The Global Challenge” (eds) R. Krishnamurthy, B. Glavovic, A. Kannen, D. Green, R. Alagappan, H. Zengcui, S. Tinti, T. Agardy. Research Publishing pp. 207-225.

Kontogianni A., Papageorgiou E., Salomatina L., Skourtos M., Zanou B., Assessing perceptions of marine environmental futures through FCM in Ukrain (forthcoming)

Kok K., (2009). The potential of Fuzzy Cognitive Maps for semi-quantitative scenario development, with an example from Brazil, Global Environmental Change vol.19, pp. 122-133.

Kosko, B. (1986). Fuzzy cognitive maps. International Journal on Man-Machine Studies, vol. 24, pp.65-75.

Kosko, B. (1992). Neural Networks and Fuzzy Systems: A Dynamical Systems Approach to Machine Intelligence. Prentice-Hall. New York.

Kouwen F. V.; P.P. Schot \& M.J. Wassen, (2008). A framework for linking advanced simulation models with interactive cognitive maps. Environmental Modeling $\mathcal{E}$ Software vol. 23 no. 9, pp. 1133-1144.

Montazemi, A.R.\& Conrath D.W. (1986) The use of cognitive mapping for information requirements analysis MIS Quarterly, March, 45-46

National Research Council (NRC). (1996). Understanding Risk: Informing Decisions in a Democratic Society. Washington, DC: National Academy Press.

Novak J. D. (1993). How do we learn our lesson? Taking students through the process. The Science Teacher 60: 50-55

OECD (2005). Evaluating Public Participation in Policy Making. Paris: OECD.

Ozesmi, U. \& S. L. Ozesmi, (2003). A participatory approach to ecosystem conservation: Fuzzy cognitive maps and stakeholder analysis in Uluabat Lake, Turkey. Environmental Management vol. 31,pp. 518-531.

Ozesmi, U. \& S. L. Ozesmi, (2004). Ecological Models based on People's Knowledge: A MultiStep Fuzzy Cognitive Mapping Approach. Ecological Modeling vol. 176,pp. 43-64.

Ozesmi, U., (1999). Modeling ecosystems from local perspectives: Fuzzy cognitive maps of the Kizilirmak Delta Wetlands in Turkey. Proceedings of 1999 World Conference on Natural Resource Modelling, Halifax, Nova Scotia, Canada

Papageorgiou E.I. \& Stylios C.D. (2008). Fuzzy Cognitive Maps, in book: Handbook of Granular Computing, editors: Witold Pedrycz, Andrzej Skowron and Vladik Kreinovich, Chapter 34, John Wiley \& Sons, Ltd, pp. 755-775. 
Papageorgiou E.I.; Markinos, Ath. \& Gemtos, Th. (2009). Application of fuzzy cognitive maps for cotton yield management in precision farming, Expert Systems with Applications, vol. 36, no. 10, pp.12399-12413.

Papageorgiou E.I.; Markinos Ath. \& Th. Gemtos, (2010). Soft Computing Technique of Fuzzy Cognitive Maps to connect yield defining parameters with yield in Cotton Crop Production in Central Greece as a basis for a decision support system for precision agriculture application, in book: Fuzzy Cognitive Maps: Advances in Theory, Methodologies, Tools, Applications, edited by M. Glykas, Springer Verlag, pp. 325-362.

Papageorgiou E.I. (2011). A new methodology for Decisions in Medical Informatics using Fuzzy Cognitive Maps based on Fuzzy Rule-Extraction techniques, Applied Soft Computing, vol. 11, pp. 500-513.

Petschel-Held G.; Block, A.; Cassel-Gintz, M.; Kropp, J.P.; Lüdeke, M.K.B.; Moldenhauer O.; Reusswig F. \& H.J. Schellnhuber, (1999). Syndromes of global change: a qualitative modeling approach to support environmental management, Environmental Modeling and Assessment vol. 4, no. 4, pp. 295-314.

Putnam, R. A. (1985). Creating facts and values. Philosophy vol. 60, pp. 187-204

Rajaram T., \& A. Das, (2009). Modeling of interactions among sustainability components of an agro-ecosystem using local knowledge through cognitive mapping and fuzzy inference system, Expert Systems with Applications, vol. 37, no. 2, pp. 1734-1744.

Ramsey D. \& Veltman, C. (2005). Predicting the effects of perturbations on ecological communities: what can qualitative models offer?Journal of Animal Ecology vol. 74, pp. 905-916.

Ramsey, D. \& Norbury, G. L. (2009). Predicting the unexpected: using a qualitative model of a New Zealand dryland ecosystem to anticipate pest management outcomes. Austral Ecology vol. 34, pp. 409-421.

Reed M. S. (2008). Stakeholder participation for environmental management: A literature review. Biological Conservation 141: 2417-2431

Reed M. S.; Graves, A. Dandy, N. Posthumus, H. Hubacek, K. Morris, J. Prell, C. Quinn, C. H. \& Stringer L. C. (2009). Who's in and why? A typology of stakeholder analysis methods for natural resource management. Journal of Environmental Management vol.90, pp.1933-1949

Robson, M. \& Kant, S., (2007). Structure of causation and its influence on cooperation: A comparative study of forest management in Ontario, Canada. Forest Policy and Economics, vol. 10(1-2), pp.70-81.

Rodriguez-Repiso, L., Setchi, R., and Salmeron, J.L. (2007). Modelling IT projects success with Fuzzy Cognitive Maps. Expert Systems with Applications, vol. 32, pp. 543-559.

Sharif, A.M. and Irani, Z. (2006). Exploring Fuzzy Cognitive Mapping for IS Evaluation. European Journal of Operational Research, vol. 173, pp.1175-1187.

Skov, F. \& J. -C. Svenning, (2003). Predicting plant species richness in a managed forest. Forest Ecology and Management vol. 6200, pp. 1-11.

W. Stach, L. A. Kurgan, and W. Pedrycz, Expert-based and Computational Methods for Developing Fuzzy Cognitive Maps, In: Glykas, M., Fuzzy Cognitive Maps: Advances in Theory, Methodologies and Applications, Springer (ISBN-10: 36-42032-19-2), 2010.

Stoney, C., \& Winstanley, D., 2001. Stakeholding: confusion or utopia? Mapping the conceptual terrain. Journal of Management Studies vol.38, pp.603-626. 
Strickert, G.E., S. Samarasinghe, \& T. Davies, (2009). Resilience models for New Zealand's Alpine Skiers based on people's knowledge and experience: a mixed method and multi-step fuzzy cognitive mapping approach, Proceedings of 18th World IMACS / MODSIM Congress, Cairns, Australia 13-17 July 2009, http://mssanz.org.au/modsim09.

Taber, W. R., (1991). Knowledge processing with fuzzy cognitive maps. Expert Systems with Applications vol. 2, pp.83-87.

Tan C. O. \& U. Ozesmi, (2006). A generic shallow lake ecosystem model based on collective expert knowledge, Hydrobiologia, vol. 563, pp.125-142.

Vliet van M.; Kok, K., Veldkamp, T. (2010). Linking stakeholders and modellers in scenario studies: The use of Fuzzy Cognitive Maps as a communication and learning tool. Futures vol. 42, no.1, pp. 1-14

Zadeh (1986). http:/ / www-bisc.cs.berkeley.edu/zadeh/papers/1986-CWW.pdf

Zhang, J., S. E. Jrgensen, C. O. Tan \& M. Beklioglu, (2003). A structurally dynamic modeling - Lake Mogan, Turkey as a case study. Ecological Modelling vol. 164, pp. 103-120.

Zorrilla P., G. Carmona, A. de la Hera, C. Varela Ortega, P. Martínez Santos (2011). Bayesian Networks as Tools for Participatory Water Resources Management: an Application to the Upper Guadiana Basin, Spain. Ecology and Society, vol. 15, no.3, [online URL: http://www.ecologyandsociety.org/vol15/iss3/art12/]. 


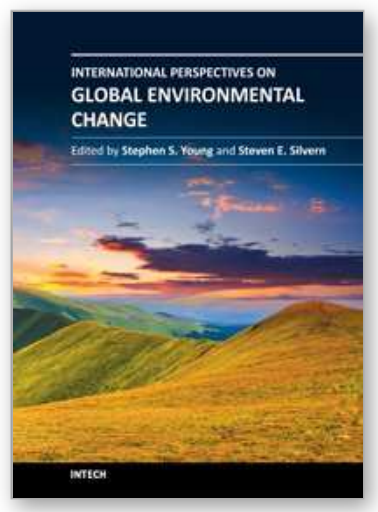

\author{
International Perspectives on Global Environmental Change \\ Edited by Dr. Stephen Young
}

ISBN 978-953-307-815-1

Hard cover, 488 pages

Publisher InTech

Published online 03, February, 2012

Published in print edition February, 2012

Environmental change is increasingly considered a critical topic for researchers across multiple disciplines, as well as policy makers throughout the world. Mounting evidence shows that environments in every part of the globe are undergoing tremendous human-induced change. Population growth, urbanization and the expansion of the global economy are putting increasing pressure on ecosystems around the planet. To understand the causes and consequences of environmental change, the contributors to this book employ spatial and nonspatial data, diverse theoretical perspectives and cutting edge research tools such as GIS, remote sensing and other relevant technologies. International Perspectives on Global Environmental Change brings together research from around the world to explore the complexities of contemporary, and historical environmental change. As an InTech open source publication current and cutting edge research methodologies and research results are quickly published for the academic policy-making communities. Dimensions of environmental change explored in this volume include: Climate change Historical environmental change Biological responses to environmental change Land use and land cover change Policy and management for environmental change

\title{
How to reference
}

In order to correctly reference this scholarly work, feel free to copy and paste the following:

Elpiniki Papageorgiou and Areti Kontogianni (2012). Using Fuzzy Cognitive Mapping in Environmental Decision Making and Management: A Methodological Primer and an Application, International Perspectives on Global Environmental Change, Dr. Stephen Young (Ed.), ISBN: 978-953-307-815-1, InTech, Available from: http://www.intechopen.com/books/international-perspectives-on-global-environmental-change/using-fuzzycognitive-mapping-in-environmental-decision-making-and-management-a-methodological-prime

\section{INTECH}

open science | open minds

\section{InTech Europe}

University Campus STeP Ri

Slavka Krautzeka 83/A

51000 Rijeka, Croatia

Phone: +385 (51) 770447

Fax: +385 (51) 686166

www.intechopen.com

\section{InTech China}

Unit 405, Office Block, Hotel Equatorial Shanghai

No.65, Yan An Road (West), Shanghai, 200040, China

中国上海市延安西路 65 号上海国际贵都大饭店办公楼 405 单元

Phone: +86-21-62489820

Fax: $+86-21-62489821$ 
(C) 2012 The Author(s). Licensee IntechOpen. This is an open access article distributed under the terms of the Creative Commons Attribution 3.0 License, which permits unrestricted use, distribution, and reproduction in any medium, provided the original work is properly cited. 